\title{
New Safety-Catch Photolabile Protecting Group
}

\author{
Emmanuel Riguet and Christian G. Bochet ${ }^{\star}$ \\ Department of Chemistry, University of Fribourg, chemin du Musée 9 , \\ CH-1700 Fribourg, Switzerland. \\ christian.bochet@unifr.ch
}

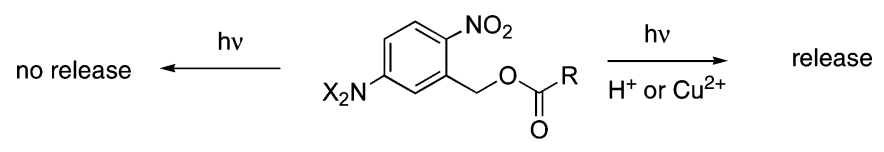

Photolabile protecting groups have proven their usefulness on many occasions. Their versions as linkers are however less attractive, as robustness and real orthogonality become critical issues. Safety-catch systems, where a preliminary activation phase is necessary, circumvent the problem of premature cleavage. In this work, we introduce a new safety-catch photolabile protecting group, whose cleavage requires the simultaneous presence of light and a chemical promoter.

Photolabile protecting groups exhibit numerous advantages over their classical chemically labile counterparts, such as the relatively soft conditions required for their deprotection and orthogonality with respect to acid- or base-sensitive groups. ${ }^{1}$ Nevertheless, to extend the scope of photolabile protecting groups, there is an important need for new methodologies to increase the selectivity of the deprotection step. Our group developed the concept of chromatic orthogonality which established that the use of monochromatic light is a powerful strategy to achieve wavelength-selective deprotection. $^{2}$

We describe here a new strategy in which the release of the protecting group requires the simultaneous presence of light and a chemical activator. It is therefore a type of safetycatch process. ${ }^{3}$ We based our work on the photochemical reactivity of ortho-nitrobenzyl derivatives such as compounds $\mathbf{1}$ and $\mathbf{2}$ which are effectively cleaved upon irradiation into amine, alcohol, or carboxylic acid, respectively (Figure 1).

(1) (a) Falvey, D. E.; Sundararajan, C. Photochem. Photobiol. Sci. 2004, 3, 831. (b) Pelliccioli, A. P.; Wirz, J. Photochem. Photobiol. Sci. 2002, 1 , 441. (c) Bochet, C. G. J. Chem. Soc., Perkin Trans. 1 2002, 125. (d) Pillai, V. N. R. Org. Photochem. 1987, 9, 225.

(2) (a) Blanc, A.; Bochet, C. G. Org. Lett. 2007, 9, 2649. (b) Blanc, A.; Bochet, C. G. J. Org. Chem. 2002, 67, 5567. (c) Bochet, C. G. Angew. Chem., Int. Ed. 2001, 40, 2071.

(3) Kenner, G. W.; McDermott, J. R.; Sheppard, R. C. Chem. Commun. 1971, 636. For a more recent overview, see: Seneci, P. Solid-Phase Synthesis and Combinatorial Technologies; John Wiley: New York, 2000; p 17.

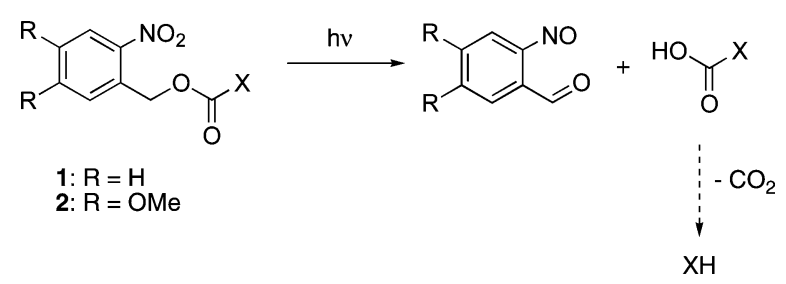

Figure 1. Photolabile derivatives of ortho-NBA.

Extensive studies on the photochemical behavior of such compounds showed that substitution on the aromatic part has an impact both on the absorption properties and on the quantum yield for photocleavage. ${ }^{4}$ Typically, in the nitroveratryloxycarbonyl (NVOC) derivatives 2, the two methoxy groups were introduced to increase the absorbance at wavelengths longer than $320 \mathrm{~nm}$. Consequently, the photolysis of NVOC derivatives 2 is possible at wavelengths longer than normally required for the NBA derivatives $1 .^{5}$ However, this modification also significantly decreased the quantum yield of the reaction. ${ }^{6}$

(4) Bochet, C. G. Tetrahedron Lett. 2000, 41, 6341 and references therein (5) Patchornik, A.; Amit, B.; Woodward, R. B. J. Am. Chem. Soc. 1970, 92,6333 .

(6) Charier, S.; Ruel, O.; Baudin, J. B.; Alcor, D.; Allemand, J. F.; Meglio, A.; Jullien, L.; Valeur, B. Chem.-Eur. J. 2006, 12, 1097-1113. 
We hypothesized that the excited state of derivatives containing an electron-releasing group para to the nitro function would have a strong charge-transfer character, which should sufficiently decrease the quantum yield to almost completely passivate the compound toward irradiation. If the electron-releasing group is an amine, we anticipated that protonation should restore the photoreactivity by cancelling the charge-transfer character in the transition state (Scheme $1)$.

Scheme 1. Inhibition of the Photoreactivity by Charge Transfer

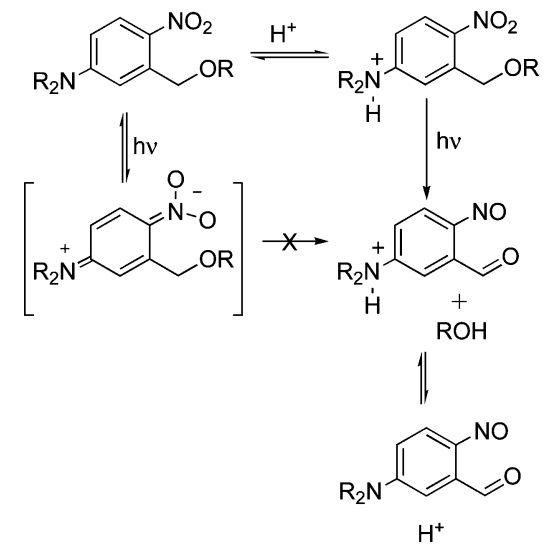

To test our hypothesis, we synthesized the esters 3-5 which contain the piperidine, morpholine, and diethyl amine subunit para to the nitro group (Figure 2).

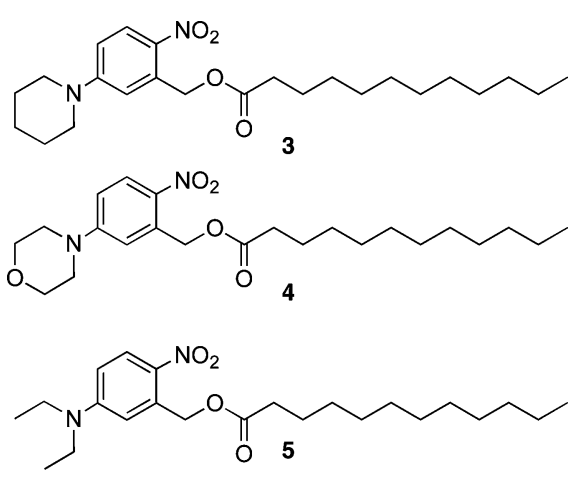

Figure 2. Safety-catch candidates.

The potentially photolabile ester $\mathbf{3}$ was prepared by simple acylation of the corresponding alcohol 10, itself prepared by nucleophilic aromatic substitution of the 4-chloro-2nitrobenzaldehyde acetal 7 with piperidine, followed by acidic hydrolysis and reduction with sodium borohydride, according to Scheme 2. Esters $\mathbf{4}$ and $\mathbf{5}$ were prepared along similar lines from alcohols $\mathbf{1 1}$ and $\mathbf{1 2}$, respectively.

We first verified that compounds $\mathbf{3 - 5}$ are inert toward irradiation at $300 \mathrm{~nm}$ in acetonitrile (Figure 3). We then
Scheme 2. Preparation of the Ester 3
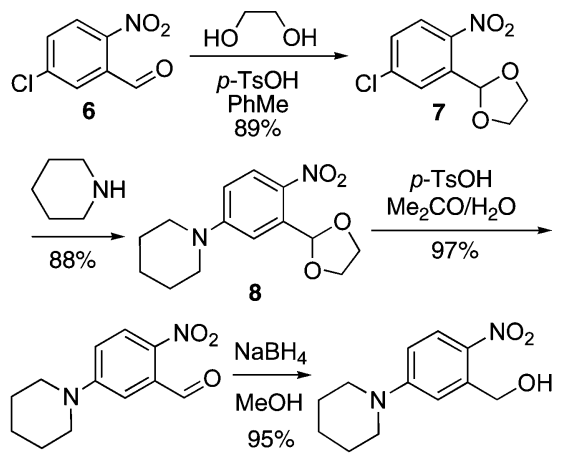

9

10

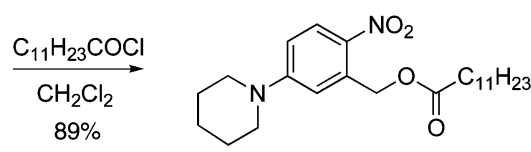

3

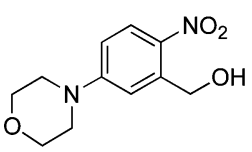

11

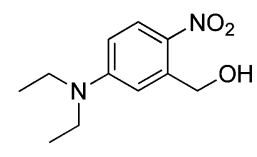

12 confirmed that protonation has the expected effect on the rate of photolysis. Indeed, in the presence of 1 equiv of

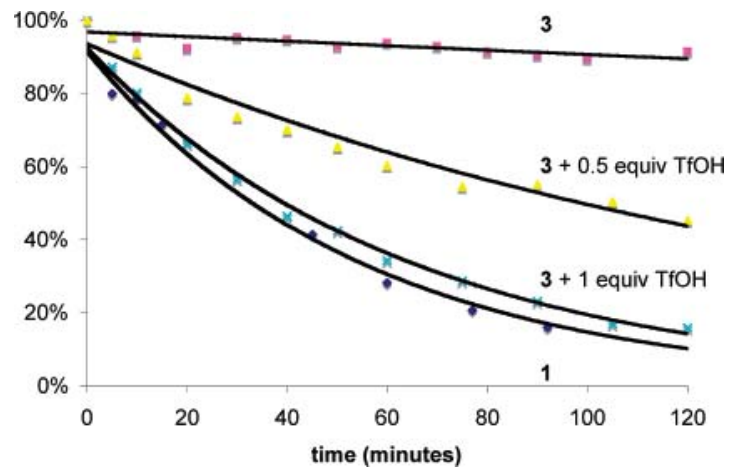

Figure 3. Photolysis of compound $\mathbf{3}$ in the presence of increasing amounts of trifluoromethane sulfonic acid. For compounds $\mathbf{4}$ and $\mathbf{5}$, see Supporting Information.

trifluoromethane sulfonic acid or 10 equiv of $\mathrm{HCl}$, the rate of photolysis is close to that of the unsubstituted NBA derivative 1 (Figure 3).

The effect of protonation is particularly visible in the UVvis spectrum of $\mathbf{3}$. The intense charge-transfer band $\left(\lambda_{\max }=\right.$ $395 \mathrm{~nm}$ ) gradually decreases upon addition of increasing amounts of a strong acid (Figure 4). Moreover, this effect is also directly observable by the disappearance of the yellow color (see pictures in the Supporting Information). The spectrocopic results might, however, not fully reflect the situation in the photolysis rate experiments because the 


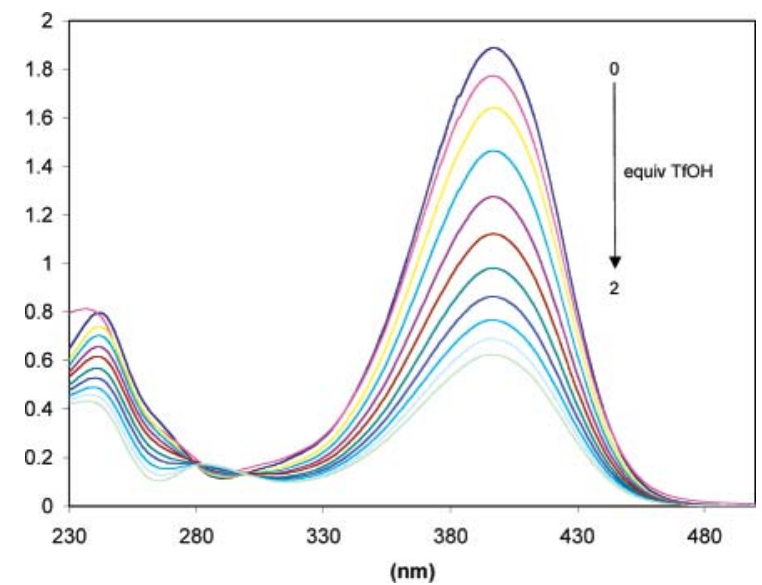

Figure 4. UV spectra of $3\left(10^{-4} \mathrm{M}\right)$ in $\mathrm{CH}_{3} \mathrm{CN}$ upon addition of trifluoromethane sulfonic acid ( $0-2$ equiv).

concentrations are very different $(0.1 \mathrm{mM}$ for the UV-vis vs $5 \mathrm{mM}$ for the photolysis). Either the protonation is nearly complete in the photolysis (as suggested by the totally colorless solution) or the fraction of reactive species is sufficient to drive the reaction to completion after reequilibration. In any case, the rates parallel the ones from NBA derivative 1 (Figure 3). It is worth pointing out that an accelerating effect of acids on NBA derivatives $\mathbf{1}$ has already been reported, but it was shown that it occurred downstream from the initial photochemical event. ${ }^{7}$ In our case, as the starting material is observed, the rates of the chemical steps following the first hydrogen abstraction are irrelevant. In the case of $\mathbf{3}$, protonation clearly leads to an acceleration of the photochemical step, i.e., the photoinduced benzylic hydrogen abstraction.

As a negative control, we verified that compound $\mathbf{3}$ did not undergo acid-catalyzed hydrolysis by adding up to 2 equiv of trifluoromethanesulfonic acid and stirring at $40{ }^{\circ} \mathrm{C}$ for $3 \mathrm{~h}$. No reaction was detected by ${ }^{1} \mathrm{H}$ NMR. To further check the compatibility with hydrolyzable functional groups (Scheme 3), we photolyzed the diester $\mathbf{1 3}$ without detecting

Scheme 3. Verification of Chemical Orthogonality

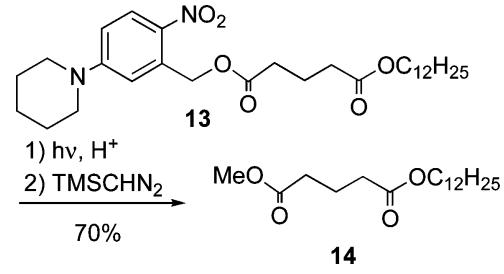

dodecanol (70\% isolated yield of $\mathbf{1 4}$, after esterification with trimethylsilyldiazomethane).

(7) (a) Hellrung, B.; Kamdzhilov, Y.; Schwörer, M.; Wirz, J. J. Am. Chem. Soc. 2005, 127, 8934. (b) Corrie, J. E. T.; Barth, A.; Munasinghe, V. R. N.; Trentham, D. R.; Hutter, M. C. J. Am. Chem. Soc. 2003, 125, 8546.
These experiments are compatible with our central hypothesis. The photoreactivity of NBA-derived esters such as $\mathbf{3}$, in which an amino group para to the nitro group quenches the photoreactivity, can be restored efficiently by a chemical activator such as a proton.

We then looked for a milder reagent to activate the photolysis. We found that $\mathrm{Cu}^{\mathrm{II}}$ could be used for this purpose. Even 1 equiv of $\mathrm{Cu}\left(\mathrm{ClO}_{4}\right)_{2}$ or $\mathrm{Cu}(\mathrm{OTf})_{2}$ almost fully restored the reactivity on 3 (Figure 5). Surprisingly, other metallic

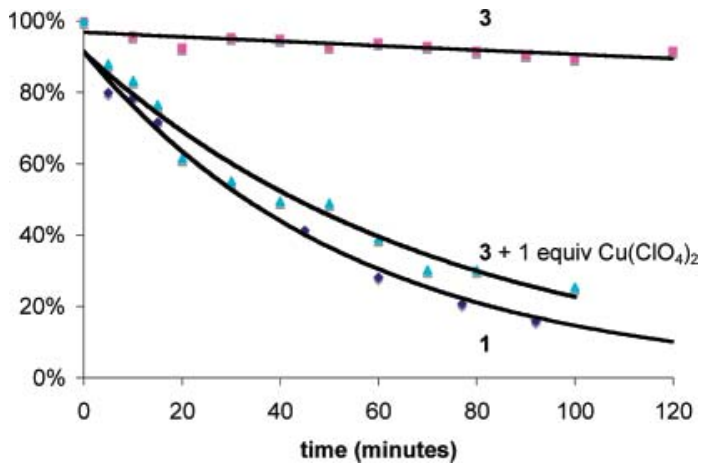

Figure 5. Photolysis of compound $\mathbf{3}$ in the presence of a copper(II) source.

cations such as $\mathrm{Zn}^{2+}$ or $\mathrm{Ni}^{2+}$ did not increase the reactivity. It is thus not ruled out that pathways more convoluted than simple complexation are involved such as, for example, single electron transfer from copper. ${ }^{8}$<smiles>O=C(O)C1CCN(c2ccc([N+](=O)[O-])c(CO)c2)CC1</smiles>

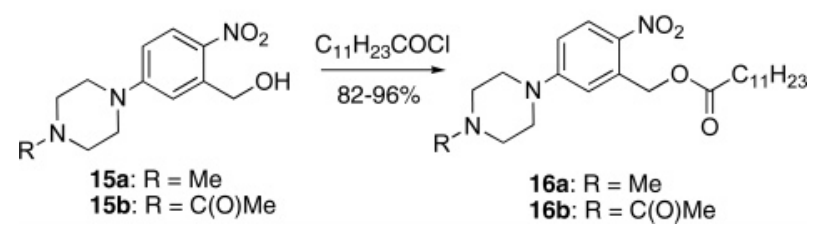

Figure 6. Further analogues of $\mathbf{3}$.

An important application of this strategy would be its use as a part of a photolabile safety-catch linker for solid-phase synthesis. ${ }^{9}$ Thus piperazine analogues such as $\mathbf{1 5 a}, \mathbf{b}$ would have both the inhibiting amine and an anchor point for a resin (Figure 6). Thus compounds such as 16a,b were prepared (see Supporting Information). Both esters were

(8) Aniline radical cations can be generated by $\mathrm{Cu}$ (II) under specific circumstances, as shown recently. See: Kirchgessner, M.; Sreenath, K.; Gopidas, K. R. J. Org. Chem. 2006, 71, 9849. 
photochemically unreactive. On the other hand, in acidic media, the reactivity of $\mathbf{1 6} \mathbf{b}$ was restored. 16a remained photoinert, probably because the protonation occurs on the unconjugated amine.

The combination of high chemical/photolytic stability and mild hydrolysis conditions makes this new photolabile protecting group an interesting platform for further optimization

(9) Efficient, safety-catch photolabile linkers have been described recently, triggered by the hydrolysis of thioketals or ketals: (a) Cano, M.; Ladlow, M.; Balasubramanian, S. J. Org. Chem. 2002, 67, 129-135. (b) Flickinger, S. T.; Patel, M.; Binkowski, B. F.; Lowe, A. M.; Li, M. H.; Kim, C.; Cerrina, F.; Belshaw, P. J. Org. Lett. 2006, 8, 2357-2360. in diverse fields of application, such as photolithography, combinatorial chemistry, or organic synthesis.

Acknowledgment. Financial support from the Swiss National Science Foundation (grant 620-066063) and the State Secretariat for Education and Research (grant C03.0025) is gratefully acknowledged. 